\title{
Comparison of long-term outcomes between radical esophagectomy and definitive chemoradiotherapy in patients with clinical T1bNOMO esophageal squamous cell carcinoma
}

\author{
Hongbo Zhao ${ }^{1,2 \#}$, Kazuo Koyanagi ${ }^{1,3}$, Ken Kato $^{4}$, Yoshinori Ito ${ }^{5}, J^{\prime}$ Itami $^{5}$, Hiroyasu Igaki $^{1}$, Yuji Tachimori ${ }^{6 \#}$ \\ ${ }^{1}$ Department of Esophageal Surgery, National Cancer Center Hospital, Tokyo, Japan; ${ }^{2}$ Department of Thoracic Surgery, National Cancer Center/ \\ National Clinical Research Center for Cancer/Cancer Hospital \& Shenzhen Hospital, Chinese Academy of Medical Science and Peking Union \\ Medical College, Shenzhen 518116, China; ${ }^{3}$ Department of Gastroenterological Surgery, Tokai University School of Medicine, Tokyo, Japan; \\ ${ }^{4}$ Department of Gastrointestinal Medical Oncology, ${ }^{5}$ Department of Radiation Oncology, National Cancer Center Hospital, Tokyo, Japan; ${ }^{6}$ Cancer \\ Care Center, Kawasaki Saiwai Hospital, Kawasaki, Japan \\ Contributions: (I) Conception and design: H Zhao, Y Tachimori, K Koyanagi; (II) Administrative support: K Koyanagi; (III) Provision of study \\ materials or patients: All authors; (IV) Collection and assembly of data: K Koyanagi, K Kato, Y Ito, J Itami, H Igaki; (V) Data analysis and \\ interpretation: H Zhao, Y Tachimori; (VI) Manuscript writing: All authors; (VII) Final approval of manuscript: All authors. \\ "These authors contributed equally to this work. \\ Correspondence to: Kazuo Koyanagi, MD, PhD. Department of Gastroenterological Surgery, Tokai University School of Medicine, 143 Shimokasuya, \\ Isehara, Kanagawa, 259-1193, Japan. Email: kkoyanagi@tsc.u-tokai.ac.jp.
}

Background: Long-term outcomes of patients with clinical T1bN0M0 thoracic esophageal squamous cell carcinoma (ESCC) treated using radical esophagectomy were compared with those treated using definitive chemoradiotherapy (dCRT).

Methods: A total of 320 consecutive patients with clinical T1bN0M0 thoracic ESCC who initially underwent radical esophagectomy or chemoradiotherapy during 2001-2011 were deemed eligible. Of these patients, 102 and 218 underwent radical esophagectomy and dCRT, respectively. Overall survival (OS) and causes of death were compared between the esophagectomy group and the chemoradiotherapy group.

Results: Five-year OS in the esophagectomy group was significantly better than that of the chemoradiotherapy group in both the overall sample and a subset of patients aged $\geq 70$ years $(\mathrm{P}=0.004$ and $\mathrm{P}=0.040)$. Male patients appeared to benefit more from radical esophagectomy $(\mathrm{P}=0.005)$. Until 2006, radical esophagectomy yielded superior results relative to dCRT $(\mathrm{P}=0.009)$. However, the survival outcomes after chemoradiotherapy were non-inferior to those after esophagectomy since 2007 ( $\mathrm{P}=0.255)$. Up to 2006, esophagectomy and chemoradiotherapy groups exhibited significant differences in the causes of death $(\mathrm{P}=0.024)$, such that the latter group had a significantly higher rate of deaths due to respiratory complications $(\mathrm{P}=0.025)$. However, the introduction of 3-dimensional radiation with CT guided planning in 2007 resolved this inter-group difference $(\mathrm{P}=0.460)$.

Conclusions: The appreciable developments in radiation technology have enabled the achievement of comparable long-term outcomes in the chemoradiotherapy group compared with the esophagectomy group in patients with clinical T1bN0M0 thoracic ESCC.

Keywords: Esophagectomy; chemoradiotherapy; esophageal squamous cell carcinoma (ESCC); outcomes

Submitted Apr 28, 2019. Accepted for publication Aug 26, 2019.

doi: $10.21037 /$ jtd.2019.10.31

View this article at: http://dx.doi.org/10.21037/jtd.2019.10.31

(C) Journal of Thoracic Disease. All rights reserved. 


\section{Introduction}

Esophageal cancer, including squamous cell carcinoma and adenocarcinoma, is the sixth cause of cancer-related mortality in the world (1). Recently, the rapid development of pretreatment diagnostic measures has increasingly led to the identification of patients with clinical stage I thoracic esophageal squamous cell carcinoma (ESCC) (7th UICCTNM classification). These stage I esophageal cancers comprise $28.4 \%$ of all esophageal cancers diagnosed in Japan (2). Generally, the reported outcomes of some treatment modalities for stage I thoracic ESCC have been favorable (2-5).

To date, radical esophagectomy with regional lymph node dissection has been main therapeutic approach for esophageal cancers, including early-stage cancer. Several reports have described favorable 5 -year overall survival (OS) rates following esophagectomy (6-8). However, radical esophagectomy is only suitable for patients who are able to tolerate this procedure. By contrast, definitive chemoradiotherapy definitive chemoradiotherapy (dCRT) enables organ preservation and a better quality of life. Chemoradiotherapy has been gradually introduced as an option for patients with locally advanced unresectable tumors and for patients with resectable tumors who had serious comorbidities or refused receiving esophagectomy. The recently published final results of the Radiation Therapy Oncology Group (RTOG) 0246 trial revealed the feasibility and encouraging long-term survival outcomes of an esophagus-preserving strategy comprising selective surgical resection after dCRT (9).

The above findings suggest that chemoradiotherapy is a promising and effective treatment for patients with early-stage ESCC $(7,9,10)$. Although chemoradiotherapy, unlike surgery, is associated with certain toxicities, its suggested advantages include esophageal preservation and the prevention of comorbidities associated with radical surgery. Accordingly, chemoradiotherapy would become standard for patients with Stage I ESCC if its efficacy were proven equivalent to that of esophagectomy. Therefore, Japan Clinical Oncology Group conducted phase II trial (JCOG9708) (11) to evaluate the safety and efficacy of concurrent chemoradiotherapy with 5 -FU plus cisplatin as a candidate test-arm regimen in a subsequent phase III trial for patients with ESCC with invasion to the submucosal layer.

In this study, we compared the outcomes between dCRT and radical esophagectomy by analyzing the OS and causes of death of both modalities in patients with clinical T1bN0M0 thoracic ESCC.

\section{Methods}

\section{Patients}

A total of 320 consecutive patients diagnosed as clinical T1bN0M0 thoracic ESCC and initially underwent radical esophagectomy or dCRT at the National Cancer Center Hospital $(\mathrm{NCCH})$ were extracted from the data base. As an initial treatment, radical esophagectomy was performed in 102 patinets and dCRT was performed in 218 patients. Forty-one patients underwent endoscopic resection (ER) for accurate staging and indication prior to undergoing radical therapies. All enrolled were clinically diagnosed with esophageal cancer classified as T1bN0M0 according to the 7 th edition of the Tumor, Node, and Metastasis system classification. The pretreatment clinical evaluations included esophageal endoscopy, endoscopic ultrasonography, computed tomography (CT) scans from cervical portion to pelvic cavity, and whole body positron emission tomography (PET) if necessary.

Patients enrolled in our study also met the following criteria: (I) histological diagnosis of squamous cell carcinoma by endoscopic biopsy; (II) primary tumor site in the thoracic esophagus; (III) no clinical evidence of lymph node or distant organ metastasis; (IV) no prior chemotherapy and/or radiotherapy; (V) adequate organ function. The CharlsonDeyo Comorbidity Index was used to assess concomitant diseases. The following exclusion criteria were also applied: (I) cervical carcinoma, (II) previous chemotherapy or radiotherapy; (III) concurrent uncontrolled illness; uncontrollable cardiac disease, hypertension or diabetes, and/or active bacterial infection. Patients who had another active synchronous cancer and died of that disease were also excluded. However, patients with other cancers that were classified as cured were deemed eligible for this study.

For each patient, the clinical stage and eligibility for radical therapy were estimated by the cancer board of NCCH. All patients consulted with both surgeons and medical oncologists. Patients who were diagnosed with tumors that were no indiction for ER because of apparent submucosal invasion were advised to undergo esophagectomy or chemoradiotherapy. The selection of therapy was determined by the patient's preference. The present study was approved by the Institutional Review Board of the NCCH (2017-061), and individual consent for 
the study was obtained from each patient.

\section{Surgical procedures}

In all cases, surgical procedures were performed by experienced esophageal surgeons. The standard procedures were esophagectomy combined with 3-field lymph node dissection and cervical anastomosis. The details of these procedures were described previously (8). Briefly, enbloc dissection of various mediastinal lymph nodes (bilateral recurrent laryngeal nerve, infra-aortic arch, subcarinal, bilateral bronchial, posterior mediastinal, and supradiaphragmatic nodes) and paraesophageal nodes was performed after mobilization of the thoracic esophagus. Patients were then turned into a spine position, and bilateral deep cervical lymph nodes (supraclavicular and cervical paraesophageal nodes) were resected. The abdominal lymph nodes (bilateral paracardial nodes, lesser curvature nodes, and along left gastric artery, common hepatic artery, splenic artery, and celiac artery nodes) were also removed. Following esophagectomy and lymphadenectomy, a gastric conduit was pulled up to the cervical portion and reconsracted.

\section{DCRT}

All patients in the chemoradiotherapy group received irradiation concurrently with 5-FU and cisplatin-based chemotherapy (11). Cisplatin was administered via slowdrip infusion; $70 \mathrm{mg} / \mathrm{m}^{2}$ on days 1 and 29 , and $5-\mathrm{FU}$ was administered via continuous infusion for $24 \mathrm{~h} ; 700 \mathrm{mg} / \mathrm{m}^{2}$ per day on days 1-4 and 29-32.

Radiation therapy was delivered in 2 Gy fractions/day on 5 days/week using megavoltage equipment (6 MV) with anterior/posterior opposed and bilateral oblique (offcord) portals. Patients received a total dose of $60 \mathrm{~Gy}$. The superior and inferior borders of the radiation field extended $3 \mathrm{~cm}$ apart from both margins of the primary tumor. The lateral, anterior, and posterior borders of the field extended $1-2 \mathrm{~cm}$ apart from the borders of the primary tumor. The clinical target volumes of the subclinical regional lymph nodes were determined according to the main location of primary tumor. If the tumor located at upper thoracic esophagus, supraclavicular, upper mediastinal, and middle mediastinal lymph nodes were included in the clinical target volumes, and if the tumor located at middle or lower thoracic esophagus, upper to lower mediastinal and perigastric lymph nodes were included. Conventional radiotherapy technology was administered until 2006, whereas 3-dimensional radiation with CT guided planning was gradually introduced to clinical practice at the $\mathrm{NCCH}$ beginning in 2007.

\section{Statistical analysis}

We analyzed cumulative OS using the Kaplan-Meier method. A Cox proportional hazards model was applied for selecting significant variables that were independently associated with OS. The criteria for the entry into the logistic regression model was determined as a valuable with a $\mathrm{P}$ valueless than 0.10 on univariate analysis. All $\mathrm{P}$ values less than 0.05 were considered to be statistically significant. All statistical analyses were performed using the SPSS software program (version 23.0; SPSS Inc., Chicago, IL, USA).

\section{Results}

\section{Patient characteristics}

A total of 320 patients with clinical T1bN0M0 thoracic ESCC (esophagectomy group, 102; chemoradiotherapy group, 218) were reviewed for this study. The median ages were 64.4 years in the esophagectomy group and 64.8 years in the chemoradiotherapy group, and the latter included significantly more patients aged $\geq 70$ years $(\mathrm{P}=0.015)$ (Table 1). No significant differences in sex, tumor site, and Charlson-Deyo score were found between the esophagectomy group and the chemoradiotherapy group.

The study period was divided into two consecutive periods, 2001-2006 and 2007-2011, to account for the substantial transition in radiation technology from conventional 2-dimensional anterior-posterior opposed fields followed by bilateral oblique boost to 3-dimensional conformal radiation therapy and fractionated stereotactic radiotherapy with CT-guided planning. No significant differences in the number of patients receiving radical esophagectomy or chemoradiotherapy were found between the two periods $(\mathrm{P}=0.282)$.

\section{Five-year OS rates}

All patients were completely followed up until death or January 2017, and the median duration of follow-up was 84 months. The 5 -year OS rate in the esophagectomy group $(88.2 \%)$ was significantly higher, when compared to the chemoradiotherapy group $(80.2 \%)(\mathrm{P}=0.004)$ (Figure 1). The 5 -year OS rates stratified by age, sex, tumor site, 
Table 1 Patients characteristics

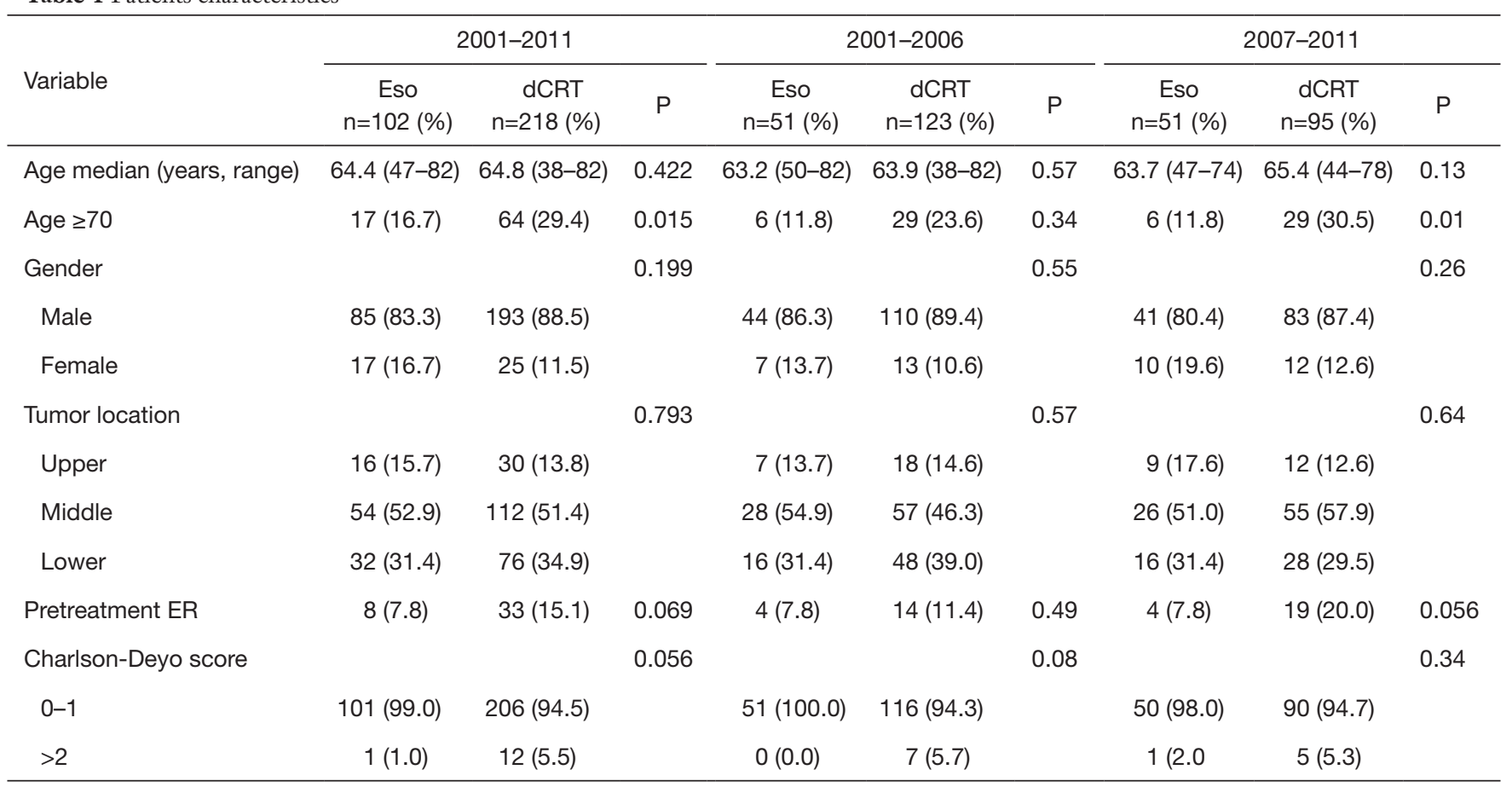

Eso, radical esophagectomy; dCRT, definitive chemoradiotherapy; ER, endoscopic resection.

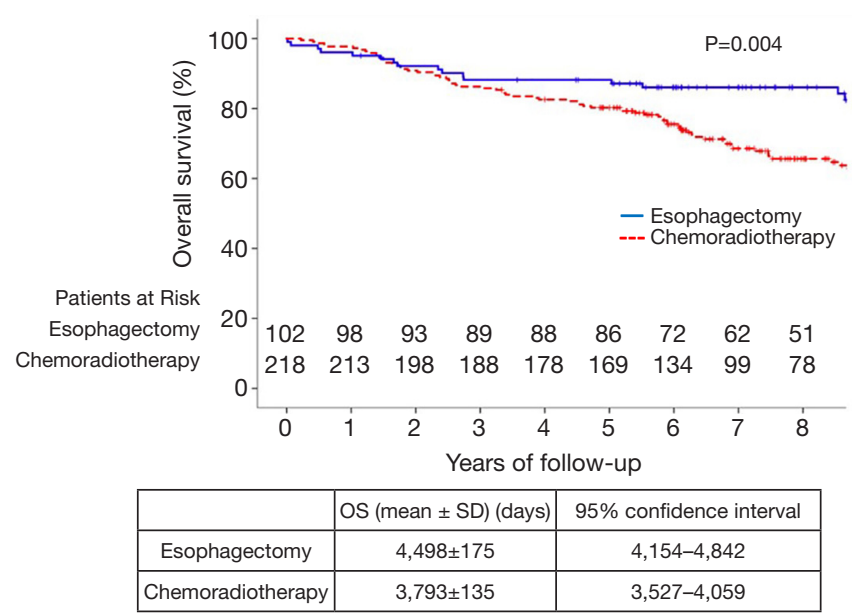

Figure 1 Overall survival curves of period 2001-2011.

and treatment period are summarized in Table 2. For patients aged $\geq 70$ years, OS was significantly higher in the esophagectomy group than in the chemoradiotherapy group $(\mathrm{P}=0.040)$. However, no significant differences were observed between these groups for patients younger than 70 years $(\mathrm{P}=0.102)$. Furthermore, radical esophagectomy yielded greater benefits than dCRT among male patients
$(\mathrm{P}=0.005)$, whereas a similar advantage was not observed for female patients $(\mathrm{P}=0.644)$. Regarding tumor location, OS was similar between the esophagectomy group and the chemoradiotherapy group for tumors at the middle or upper esophagus $(\mathrm{P}>0.05)$, whereas surgery yielded significantly better OS for tumors at the lower esophagus $(\mathrm{P}=0.005)$.

Figure 2 presents the OS curves during 2001-2006 and 2007-2011. The corresponding 5-year OS rates in the former period were $86.3 \%$ and $75.6 \%$ in the esophagectomy and chemoradiotherapy groups, respectively. On the other hand, those in the latter period were $90.1 \%$ and $86.2 \%$ in the esophagectomy and chemoradiotherapy groups, respectively. Before 2007, radical esophagectomy conferred greater benefits than dCRT $(\mathrm{P}=0.009)$. However, neither treatment option exhibited an overwhelming advantage over the another after $2007(\mathrm{P}=0.255)$.

\section{Causes of death and respiratory complications}

Nineteen (18.6\%) and 76 (34.9\%) deaths were recorded among patients in the esophagectomy and chemoradiotherapy groups, respectively. The causes of death were divided into three categories: primary esophageal cancer, other cancers, and other diseases (Table 3). Respiratory complications, 
including late toxicities of chemoradiotherapy, were classified as other diseases. The esophagectomy and chemoradiotherapy groups differed significantly in terms of the causes of death $(\mathrm{P}=0.024)$. Specifically, the chemoradiotherapy group included a significantly higher number of deaths due to other diseases, including respiratory complications.

Respiratory complications associated with esophagectomy

Table 2 Univariate analysis of 5-year overall survival

\begin{tabular}{|c|c|c|c|}
\hline \multirow{2}{*}{ Variable } & \multicolumn{3}{|c|}{ 5-year overall survival rate } \\
\hline & Eso (\%) & dCRT (\%) & $\mathrm{P}$ \\
\hline \multicolumn{4}{|l|}{ Age } \\
\hline$<70$ & 88.2 & 83.8 & 0.102 \\
\hline$\geq 70$ & 87.8 & 71.6 & 0.040 \\
\hline \multicolumn{4}{|l|}{ Sex } \\
\hline Male & 89.3 & 79.2 & 0.005 \\
\hline Female & 83.3 & 89.2 & 0.644 \\
\hline \multicolumn{4}{|l|}{ Tumor location } \\
\hline Upper & 80.4 & 80.0 & 0.771 \\
\hline Middle & 88.9 & 83.0 & 0.122 \\
\hline Lower & 90.6 & 76.2 & 0.005 \\
\hline \multicolumn{4}{|c|}{ Treatment period } \\
\hline 2001-2011 & 88.2 & 80.2 & 0.004 \\
\hline 2001-2006 & 86.3 & 75.6 & 0.009 \\
\hline 2007-2011 & 90.1 & 86.2 & 0.255 \\
\hline
\end{tabular}

Eso, radical esophagectomy; dCRT, definitive chemoradiotherapy. (e.g., pleural effusion, embolism, and pneumonitis) and chemoradiotherapy (e.g., pericardial effusion, radiation pneumonitis) can strongly affect post-treatment and long-term morbidity and mortality in esophageal cancer patients. Therefore, we compared deaths due to respiratory complications between the esophagectomy and chemoradiotherapy groups and found a significantly higher incidence of respiratory complications in the chemoradiotherapy group $(\mathrm{P}=0.014)$. Particularly, in the period before 2007, the incidence of death due to respiratory complications in the chemoradiotherapy group was significantly higher than that of the esophagectomy group $(\mathrm{P}=0.025)$, however, this significance was not found after $2007(\mathrm{P}=0.462)$.

\section{Cox proportional hazards model}

A Cox proportional hazards model identified an age $\geq 70$ years and radical esophagectomy during 2001-2011 and 2001-2006 as independent factors that associated with an increased OS rate (Table 4). Notably, none of those factors significantly affect the likelihood of OS during 2007-2011.

\section{Discussion}

ESCCs and adenocarcinomas traditionally have been considered a single entity with similar treatment recommendations. However, clinicians have long realized that these malignancies behave differently, particularly in terms of responsiveness to chemoradiotherapy (12). To date, chemoradiotherapy has been considered a curative treatment option that confers substantial improvements in

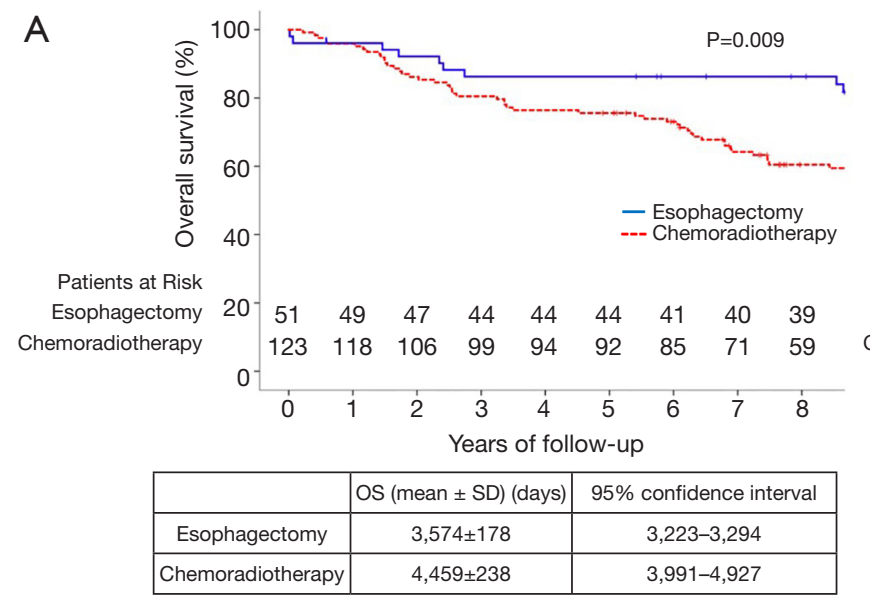

B
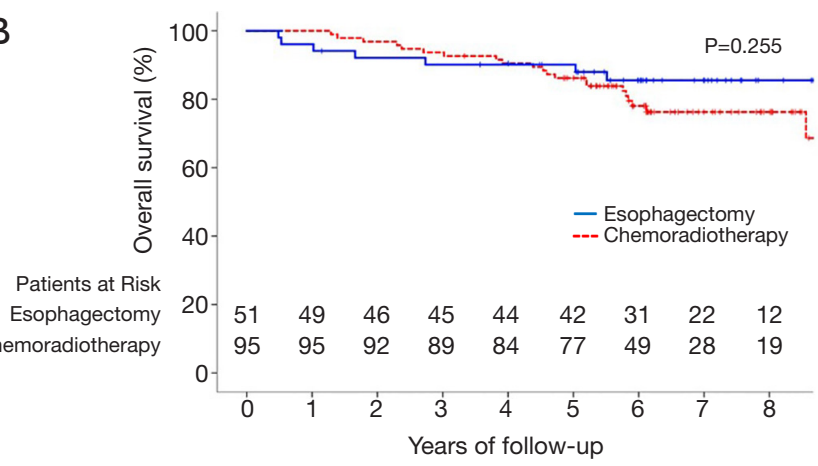

\begin{tabular}{|c|c|c|}
\hline & OS (mean \pm SD) (days) & 95\% confidence interval \\
\hline Esophagectomy & $3,030 \pm 98$ & $2,834-3,222$ \\
\hline Chemoradiotherapy & $3,212 \pm 139$ & $2,939-3,483$ \\
\hline
\end{tabular}

Figure 2 Overall survival curves of period 2001-2006 (A) and 2007-2011 (B). 
survival relative to radical esophagectomy for ESCC (8$11,13-15)$. This was the largest cohort study to compare the relatively long-term survival outcomes between radical esophagectomy and dCRT in patients with superficial thoracic ESCC that involved no clinical lymph node metastasis. Specifically, complete follow-up data for up to 10 years were available for all patients in our study.

Consistent with our previous surgical study, we have demonstrated significantly better 5 -year OS rates with radical esophagectomy than with chemoradiotherapy in patients with thoracic ESCC diagnosed with clinical T1bN0M0 (8). Several studies have revealed the effectiveness of chemoradiotherapy for the treatment of stage I ESCC, including clinical T1bN0M0 (13). For example, a Phase II trial (JCOG9708) by Kato et al. (11) reported 4-year survival outcomes after chemoradiotherapy that were equivalent to our previous surgical results.

To further explore potential differences in our results, the study period was divided into two consecutive periods

Table 3 Causes of death

\begin{tabular}{lccc}
\hline Variable & Eso $n=102(\%)$ & dCRT $n=218(\%)$ & $P$ \\
\hline Causes of death & & 0.024 \\
Esophageal cancer & $8(7.8)$ & $27(12.4)$ & \\
Other cancer & $2(2.0)$ & $15(6.9)$ & \\
Other disease & $9(8.8)$ & $34(15.6)$ & \\
Respiratory complications & & & \\
2001-2011 & $1(1.0)$ & $17(7.8)$ & 0.014 \\
$2001-2006$ & $1(2.0)$ & $16(7.3)$ & 0.025 \\
$2007-2011$ & $0(0.0)$ & $1(0.5)$ & 0.462 \\
\hline
\end{tabular}

Eso, radical esophagectomy; dCRT, definitive chemoradiotherapy.
(2001-2006 and 2007-2011) to account for the appreciable development of radiation technology around 2007. A subsequent analysis revealed that the survival advantages conferred by esophagectomy over chemoradiotherapy prior to 2007 disappeared after 2007. As noted previously, 2007 was the year when the widely used conventional protocol (2-dimensional anterior-posterior opposed fields followed by bilateral oblique boost) shifted to 3-dimensional conformal radiation therapy with CT-guided planning and fractionated stereotactic radiotherapy technology at our institution. In a study of patients with clinical T1bN0M0 ESCC who were treated between 1995 and 2007, Motoori et al. (14) reported that OS of the surgically treated patients did not differ from that of the patients treated by conventional radiotherapy combined with concurrent chemotherapy. However, esophagectomy yielded significantly better progression-free survival, compared to chemoradiotherapy. By contrast, Murakami et al. (15) reported that organpreserving chemoradiotherapy yielded a high rate of locoregional control and favorable overall 5-year survival (86\%) in patients with clinical T1bN0M0 ESCC. That study included patients recruited from 2001 to 2011, and all patients underwent 3-dimensional radiotherapy treatment planning. The reported late toxicities and higher mortality rate associated with conventional radiotherapy might be attributable to an extended field of irradiation (16).

Our results also revealed similar rates of failures and salvage therapy after dCRT in patients treated before and after 2007. Compared with conventional radiotherapy, the newer radiotherapy technology enabled better targeting of tumors while reducing the irradiated volumes of normal tissue (17). Given the appreciable development of radiation technology, dCRT could potentially be used to achieve long-term outcomes comparable to those of radical esophagectomy in patients with clinical T1bN0M0 thoracic

Table 4 Multivariate Cox proportional hazards analysis

\begin{tabular}{|c|c|c|c|c|c|c|c|c|c|}
\hline Variable & \multicolumn{3}{|c|}{ 2001-2011 } & \multicolumn{3}{|c|}{ 2001-2006 } & \multicolumn{3}{|c|}{ 2007-2011 } \\
\hline Age & 0.54 & $0.35-0.84$ & 0.005 & 0.39 & $0.24-0.63$ & $<0.001$ & 1.35 & $0.53-3.39$ & 0.53 \\
\hline Gender & 0.69 & $0.35-1.37$ & 0.29 & 0.52 & $0.21-1.31$ & 0.17 & 1.09 & $0.38-3.16$ & 0.87 \\
\hline Location & 0.81 & $0.53-1.23$ & 0.32 & 1.06 & $0.61-1.66$ & 0.98 & 2.16 & $1.00-4.64$ & 0.048 \\
\hline Period & 1.50 & $0.95-2.40$ & 0.08 & & & & & & \\
\hline
\end{tabular}

Age, age $\geq 70$ vs. age <70; gender, male vs. female; location, low thoracic tumor vs. other thoracic tumor; therapeutic strategy, radical esophagectomy vs. definitive chemoradiotherapy; period, treatment period, 2001-2006 vs. 2007-2011. HR, hazard ratio. 
ESCC. Our results confirm this result of developments in radiotherapy technology.

Furthermore, we analyzed the causes of death in the two treatment groups. Notably, deaths due to primary esophageal cancer, other cancers, and other causes occurred significantly more frequently after dCRT than after radical esophagectomy. First, deaths due to other diseases occurred almost twice as frequently in the chemoradiotherapy group than in the esophagectomy group. A further analysis found that the chemoradiotherapy group showed a significantly higher rate of respiratory complications, including lateset pleural effusion, pericardial effusion, and radiationinduced pneumonitis, compared to the esophagectomy group. Late toxicity after chemoradiotherapy is a wellknown and sometimes fatal complication that may affect patients even years after achieving a complete response (18). Kato et al. (19) reported that up to $5.3 \%$ of the patients who underwent chemoradiotherapy may have died of late toxicity even after the completion of the chemoradiotherapy. Murakami et al. (15) reported the life-threating delayed toxicities (more than grade 3), such as pleural effusion, pericardial effusion, and radiation pneumonitis. The most recent study revealed that $12.1 \%$ of patients who received chemoradiotherapy via a conventional method experienced late toxicities (20). Additionally, we found that deaths due to respiratory complications occurred more frequently in the chemoradiotherapy group than in the esophagectomy group before 2007, but not after 2007. This discrepancy supports the view that considerable advances in radiotherapy technology have improved the safety of chemoradiotherapy for the treatment of ESCC.

Second, relatively higher number of patients in the chemoradiotherapy groups died due to primary esophageal cancer. In contrast to esophagectomy, esophageal failure is more likely to occur because the esophagus itself is preserved during and after chemoradiotherapy. Yamamoto et al. (13) reported a significantly higher incidence of local recurrence after chemoradiotherapy relative to esophagectomy in a study of patients with clinical stage I ESCC. Motoori et al. (14) also reported that both local and lymph node recurrences occurred more frequent in the chemoradiotherapy group, whereas the incidence of distant recurrence in the chemoradiotherapy group was not different from the esophagectomy group. Moreover, the rate of local recurrence after chemoradiotherapy might increase with longer follow-up. Although early-stage patients may be expected to achieve a favorable long-term survival, recurrences of primary tumor, regional lymph nodes, and metastases to distant organs are important issues requiring further attention.

Third, we found that deaths due to other cancers were more than three times more frequent in the chemoradiotherapy group than in the esophagectomy group. Obviously, the chemoradiotherapy group contained a significantly higher frequency of patients aged $\geq 70$ years, compared to the esophagectomy group. Cancer is an agerelated disease, as the risk of cancer increases with aging (21). Matsubara et al. reported that the risk of secondary cancer after esophageal cancer increased over time even if the patients survived from esophageal cancer (22). Additionally, chemoradiotherapy may increase the risk of developing a secondary cancer in the radiated field (23). In a phase II trial of chemoradiotherapy for stage I ESCC, Kato et al. found that $25 \%$ of patients developed a second primary cancer in another organ after chemoradiotherapy (11).

Our Cox proportional hazards model identified an age $\geq 70$ years and radical esophagectomy as independent prognostic factors associated with increased OS during both 2001-2011 and 2001-2006. By contrast, neither factor significantly affected the OS outcomes during 20072011. Previous studies identified lymph node metastasis, undifferentiations, length of the tumors, and vessel invasions as prognostic factors that significantly associated with OS in patients with submucosal esophageal cancer (24-26). In the study, these factors could not be associated with the OS. Pre ER also did not affect the OS rates. On the other hand, we clearly demonstrated the comparable improvements in radiation technology for the patients with ESCC according to the period in the study.

This study had several limitations of note. This was a non randomized and retrospective analysis of data from a single institution. The therapeutic strategies applied to each patient varied somewhat over time, and new techniques and new treatment concepts were applied and accepted gradually. Selection bias may have existed before treatment. Larger studies are needed to fully assess these outcomes. Currently, the JCOG0502 trial, a prospective multicenter phase III study that aims to identify additional predictive and prognostic factors in patients with clinical T1bN0M0 thoracic ESCC, has been conducting to compare the OS between esophagectomy and chemoradiotherapy and we are expecting the result from multicenter trial.

\section{Conclusions}

In conclusion, the appreciable advances in radiation 
technology have enabled comparative long-term outcomes between dCRT and radical esophagectomy in patients with clinical T1bN0M0 thoracic ESCC.

\section{Acknowledgments}

None.

\section{Footnote}

Conflicts of Interest: The authors have no conflicts of interest to declare.

Ethical Statement: The authors are accountable for all aspects of the work in ensuring that questions related to the accuracy or integrity of any part of the work are appropriately investigated and resolved. The present study was approved by the Institutional Review Board of the NCCH (2017-061), and individual consent for the study was obtained from each patient.

\section{References}

1. Torre LA, Bray F, Siegel RL, et al. Global cancer statistics, 2012. CA Cancer J Clin 2015;65:87-108.

2. Tachimori Y, Ozawa S, Numasaki H, et al. Comprehensive Registry of Esophageal Cancer in Japan, 2009. Esophagus 2016;13:110-37.

3. Hölscher AH, Bollschweiler E, Schroder W, et al. Prognostic impact of upper, middle, and lower third mucosal or submucosal infiltration in early esophageal cancer. Ann Surg 2011;254:802-7; discussion 807-8.

4. Kurokawa Y, Muto M, Minashi K, et al. A phase II trial of combined treatment of endoscopic mucosal resection and chemoradiotherapy for clinical stage I esophageal carcinoma: Japan Clinical Oncology Group Study JCOG0508. Jpn J Clin Oncol 2009;39:686-9.

5. Hayashi $Y$, Nishida T, Tsujii $M$, et al. Lymph node enlargement after definitive chemoradiotherapy for clinical stage I esophageal squamous cell carcinoma. BMC Cancer 2014;14:706.

6. Tachibana M, Yoshimura H, Kinugasa S, et al. Clinicopathological features of superficial squamous cell carcinoma of the esophagus. Am J Surg 1997;174:49-53.

7. Takahashi H, Arimura Y, Masao H, et al. Endoscopic submucosal dissection is superior to conventional endoscopic resection as a curative treatment for early squamous cell carcinoma of the esophagus (with video).
Gastrointest Endosc 2010;72:255-64, 264.e1-2.

8. Igaki H, Kato H, Tachimori Y, et al. Clinicopathologic characteristics and survival of patients with clinical Stage I squamous cell carcinomas of the thoracic esophagus treated with three-field lymph node dissection. Eur J Cardiothorac Surg 2001;20:1089-94.

9. Swisher SG, Moughan J, Komaki RU, et al. Final Results of NRG Oncology RTOG 0246: An Organ-Preserving Selective Resection Strategy in Esophageal Cancer Patients Treated with Definitive Chemoradiation. J Thorac Oncol 2017;12:368-74.

10. Stahl M, Stuschke M, Lehmann N, et al. Chemoradiation with and without surgery in patients with locally advanced squamous cell carcinoma of the esophagus. J Clin Oncol 2005;23:2310-7.

11. Kato H, Sato A, Fukuda H, et al. A phase II trial of chemoradiotherapy for stage I esophageal squamous cell carcinoma: Japan Clinical Oncology Group Study (JCOG9708). Jpn J Clin Oncol 2009;39:638-43.

12. Lagisetty KH, Beer DG, Chang AC. Squamous cell carcinomas and adenocarcinomas of the esophagus: One treatment does not rule them all. J Thorac Cardiovasc Surg 2017;154:1446-7.

13. Yamamoto $\mathrm{S}$, Ishihara R, Motoori $M$, et al. Comparison between definitive chemoradiotherapy and esophagectomy in patients with clinical stage I esophageal squamous cell carcinoma. Am J Gastroenterol 2011;106:1048-54.

14. Motoori M, Yano M, Ishihara R, et al. Comparison between radical esophagectomy and definitive chemoradiotherapy in patients with clinical T1bN0M0 esophageal cancer. Ann Surg Oncol 2012;19:2135-41.

15. Murakami Y, Takahashi I, Nishibuchi I, et al. Long-term results of definitive concurrent chemoradiotherapy for patients with esophageal submucosal cancer (T1bN0M0). Int J Clin Oncol 2015;20:897-904.

16. Sasaki $Y$, Kato K. Chemoradiotherapy for esophageal squamous cell cancer. Jpn J Clin Oncol 2016;46:805-10.

17. Ozyigit G, Gultekin M. Current role of modern radiotherapy techniques in the management of breast cancer. World J Clin Oncol 2014;5:425-39.

18. Ishikura S, Nihei K, Ohtsu A, et al. Long-term toxicity after definitive chemoradiotherapy for squamous cell carcinoma of the thoracic esophagus. J Clin Oncol 2003;21:2697-702.

19. Kato K, Muro K, Minashi K, et al. Phase II study of chemoradiotherapy with 5-fluorouracil and cisplatin for Stage II-III esophageal squamous cell carcinoma: JCOG trial (JCOG 9906). Int J Radiat Oncol Biol Phys 
2011;81:684-90.

20. Ito H, Itasaka S, Sakanaka K, et al. Long-term complications of definitive chemoradiotherapy for esophageal cancer using the classical method. J Radiat Res 2017;58:106-13.

21. Ershler WB, Longo DL. Aging and cancer: issues of basic and clinical science. J Natl Cancer Inst 1997;89:1489-97.

22. Kumagai Y, Kawano T, Nakajima Y, et al. Multiple primary cancers associated with esophageal carcinoma. Surg Today 2001;31:872-6.

23. Boffetta P, Kaldor JM. Secondary malignancies following

Cite this article as: Zhao $\mathrm{H}$, Koyanagi $\mathrm{K}$, Kato $\mathrm{K}$, Ito $\mathrm{Y}$, Itami J, Igaki H, Tachimori Y. Comparison of long-term outcomes between radical esophagectomy and definitive chemoradiotherapy in patients with clinical T1bN0M0 esophageal squamous cell carcinoma. J Thorac Dis 2019;11(11):4654-4662. doi: 10.21037/jtd.2019.10.31 cancer chemotherapy. Acta Oncol 1994;33:591-8.

24. Grotenhuis BA, van Heijl M, Zehetner J, et al. Surgical management of submucosal esophageal cancer: extended or regional lymphadenectomy? Ann Surg 2010;252:823-30.

25. Tajima Y, Nakanishi Y, Ochiai A, et al. Histopathologic findings predicting lymph node metastasis and prognosis of patients with superficial esophageal carcinoma: analysis of 240 surgically resected tumors. Cancer 2000;88:1285-93.

26. Natsugoe S, Matsumoto M, Okumura H, et al. Prognostic factors in patients with submucosal esophageal cancer. J Gastrointest Surg 2004;8:631-5. 